\title{
COEFICIENTE DE CULTURA DA LIMA-ÁCIDA TAHITI NO OUTONO-INVERNO DETERMINADO POR LISIMETRIA DE PESAGEM EM PIRACICABA - SP
}

\author{
CÍCERO R. A. BARBOZA JÚNIOR ${ }^{1}$, MARCOS V. FOLEGATTI ${ }^{2}$, FÁBIO J. ROCHA ${ }^{3}$, \\ ROBERTO T. ATARASSI ${ }^{4}$
}

RESUMO: O Brasil é o maior produtor mundial de citros, com destaque para o Estado de São Paulo, maior produtor nacional. Recentes estudos mostram que a área irrigada de citros em São Paulo tem aumentado significativamente nos últimos anos. Porém, a falta de informações sobre o manejo eficiente da irrigação na cultura de citros é uma das principais dificuldades enfrentadas pelos produtores. Com o intuito de atender a essa necessidade, este trabalho teve como objetivos determinar a evapotranspiração de uma planta adulta de limeira-ácida 'Tahiti' (Citrus latifolia Tan.) e o coeficiente de cultivo $(\mathrm{Kc})$ no período seco (outono-inverno), utilizando a técnica de lisimetria de pesagem. O experimento foi realizado na ESALQ/USP em Piracicaba - SP, em área irrigada por gotejamento, com plantas espaçadas de $7 \times 4 \mathrm{~m}$, sendo cada planta atendida por quatro pontos de molhamento no solo, distribuídos de forma equidistantes entre si. Foi realizado o monitoramento climático, utilizando estação meteorológica automatizada, e a determinação da evapotranspiração da cultura por lisímetro de pesagem. Durante o período de estudo, o Kc variou entre 0,82 e 1,18, e a ETc variou entre 1,2 e 5,6.

PALAVRAS-CHAVE: irrigação localizada, evapotranspiração da cultura, coeficiente de cultivo.

\section{CROP COEFFICIENT OF ACID LIME TAHITI DURING AUTUMN-WINTER PERIOD DETERMINED BY WEIGHING LYSIMETER TECHNIQUE IN PIRACICABA - SP, BRAZIL}

\begin{abstract}
Brazil is the largest world producer of citrus crop, with São Paulo state leading as the largest national producer. Recent studies show that irrigated areas of citrus in the state have been increasing significantly in the last few years. However, lack of information on irrigation management related to this crop is one of the main problems encountered by the farmers. In order to help solve the above problem, the objective of this work was to determine the evapotranspiration of acid lime adult plant variety 'Tahiti' (Citrus latifolia Tan.) and the crop coefficient during dry period (autumn - winter) using a weighing lysimeter technique. The experiment was carried out at ESALQ/USP in Piracicaba - SP, Brazil, in a drip irrigated area with plant spacing of $7 \times 4 \mathrm{~m}$. Each plant was supplied with four wetting equidistant emitters. The climatic data was obtained by the use of automated meteorological station and the crop evapotraspiration by the use of a weighting lysimeter. During the study period, Kc varied from 0.82 to 1.18, and Etc varied from 1.20 to 5.6.
\end{abstract}

KEYWORDS: drip irrigation, crop evapotraspiration, crop coefficient.

\section{INTRODUÇÃO}

A lima-ácida 'Tahiti' (Citrus latifolia Tanaka), conhecida e consagrada entre os consumidores brasileiros como limão Tahiti, é uma das preciosidades da citricultura. É uma espécie americana de citro, pois sua origem é a Califórnia, Estados Unidos, onde surgiu a partir de sementes de limão introduzidas do Tahiti, por volta de 1870. Sua cultura difundiu-se pelos países das três Américas, único continente onde o limão Tahiti é produzido comercialmente. A produção de lima-ácida 'Tahiti' (Citrus latifolia Tan.) vem crescendo e ocupando lugar cada vez mais importante na

\footnotetext{
${ }^{1}$ Doutorando em Agronomia (Irrigação e Drenagem), Departamento de Engenharia Rural, ESALQ/USP, Piracicaba - SP, Fone : (0XX19) 3447.8554, crabjuni@esalq.usp.br

${ }^{2}$ Professor Titular, Departamento de Engenharia Rural, ESALQ/USP, Piracicaba - SP.

${ }^{3}$ Doutorando em Agronomia (Irrigação e Drenagem), Departamento de Engenharia Rural, ESALQ/USP, Piracicaba - SP

${ }^{4}$ Pós-Doutorando em Agronomia (Irrigação e Drenagem), Departamento de Engenharia Rural, ESALQ/USP, Piracicaba - SP.

Recebido pelo Conselho Editorial em: 13-11-2007
}

Aprovado pelo Conselho Editorial em: 21-1-2009

Eng. Agríc., Jaboticabal, v.28, n.4, p.691-698, out./dez. 2008 
citricultura brasileira, com sua exportação em ascensão, além do consumo no mercado interno (BARROS et al., 1991).

O conhecimento da evapotranspiração da cultura (ETc) e do coeficiente de cultivo (Kc) é fundamental para a outorga de água, para dimensionar e manejar a irrigação de uma cultura, uma vez que a demanda hídrica da planta varia conforme a radiação solar incidente no local, tipo de solo, variedade e idade da planta. Entretanto, a grande maioria das informações quanto ao consumo hídrico das plantas cítricas, utilizando-se de lisímetros, provém de países de regime climático diferente do que ocorre no Brasil (BOMAN,1994; CASTEL, 1994; YANG et al., 2003).

$\mathrm{O}$ valor de Kc muda de acordo com o crescimento e o desenvolvimento da cultura, variando também com a fração de cobertura da superfície do solo pela vegetação à medida que as plantas envelhecem e atingem a maturação (SEDIYAMA et al., 1998).

Diversos autores reportam o uso de lisímetros de pesagem como ferramenta-padrão em estudos de consumo de água das culturas, seja na determinação da evapotranspiração, seja na calibração de modelos agrometeorológicos de estimativa (HOWELL et al., 1991; SILVA et al., 1999; CAMPECHE, 2002).

É de fundamental importância a obtenção de valores de Kc que possam ser utilizados para o dimensionamento e o manejo de sistemas de irrigação no Brasil. Assim, este estudo teve como objetivo determinar a evapotranspiração de uma planta adulta de limeira-ácida 'Tahiti' e estimar o coeficiente de cultivo durante o período de outono/inverno (período seco), usando a técnica da lisimetria de pesagem.

\section{MATERIAL E MÉTODOS}

O experimento foi conduzido na área experimental do Departamento de Engenharia Rural, da ESALQ-USP, situada no município de Piracicaba - SP, nas seguintes coordenadas geográficas: $22^{\circ} 41^{\prime} 58^{\prime}$ ' de latitude sul; 47³8'42'" de longitude oeste e aproximadamente $511 \mathrm{~m}$ de altitude.

O experimento foi conduzido de abril (outono) a setembro (final do inverno) de 2007. A temperatura, a umidade relativa média e o total de precipitação durante a condução do experimento foram de $19,6^{\circ} \mathrm{C}, 72,5 \%$ e $336,8 \mathrm{~mm}$, respectivamente.

O solo da área experimental é classificado como Nitossolo Vermelho (EMBRAPA, 1999), textura argilosa, com densidade média entre a superfície e $1 \mathrm{~m}$ de profundidade de $1,3 \mathrm{~kg} \mathrm{dm}^{-3} \mathrm{e}$ com 5\% de declividade média. A saturação por bases na camada de 0 a $0,6 \mathrm{~m}$ de profundidade era de aproximadamente $64 \%$ e pH médio de 5,2. A adubação da área foi feita seguindo recomendação de RAIJ et al. (1992).

O estudo foi conduzido com plantas de lima-ácida 'Tahiti' [Citrus latifolia (Yu. Tanaka) Tanaka cv. IAC 5], enxertada em citrumelo 'Swingle' [Poncirus trifoliata (L.) Raf. $\times$ Citrus paradisi Macf.]. O pomar, instalado em área de aproximadamente 1,0 ha, com plantas espaçadas de 4 x 7 m, foi implantado na primeira quinzena de maio de 2001, totalizando 360 plantas por hectare.

\section{Sistema de irrigação}

Utilizou-se de sistema de irrigação por gotejamento no pomar, dotado de emissores autocompensantes (Modelo PCJ, Netafim off-line), com vazão de $4 \mathrm{~L} \mathrm{~h}^{-1}$ cada. Foram utilizados quatro emissores por planta, os quais foram distribuídos de forma equidistantes entre si e localizados a $0,5 \mathrm{~m}$ do tronco, sendo a vazão total do sistema de $5.760 \mathrm{~L} \mathrm{~h}^{-1}$. A uniformidade de distribuição de água do sistema foi avaliada e apresentou valor de $95 \%$. A umidade do solo foi mantida entre 0,35 e $0,40 \mathrm{~m}^{3} \mathrm{~m}^{-3}$, em turno de rega que variou de 1 a 4 dias para reposição da água evapotranspirada. 


\section{Evapotranspiração}

Neste estudo, para a determinação da evapotranspiração da cultura (ETc), foi utilizado um lisímetro de pesagem com três células de carga eletrônicas acopladas a um sistema automático de coleta de dados (datalloger CR23X, da Campbell Scientific, Logan, Utah, EUA). O lisímetro localizava-se próximo ao centro da área experimental, contendo uma planta com características semelhantes (Tabela 1) e sob o mesmo sistema de manejo das demais plantas do pomar, com reposição de $100 \%$ da água evapotranspirada pelo sistema. O lisímetro apresentava as dimensões de 4,0 $\mathrm{m}$ de diâmetro e $1,3 \mathrm{~m}$ de profundidade com sensibilidade de $0,45 \mathrm{~kg}$, correspondendo a $0,036 \mathrm{~mm}$ e exatidão de $0,1 \mathrm{~mm}$ (Figura $1 \mathrm{~b}$ ). Maiores detalhes sobre a construção e a calibração desse equipamento podem ser obtidos em CAMPECHE (2002). Na Figura 1a, pode ser vista a estação automática utilizada para a determinação da evapotranspiração de referência e armazenamento de dados meteorológicos.

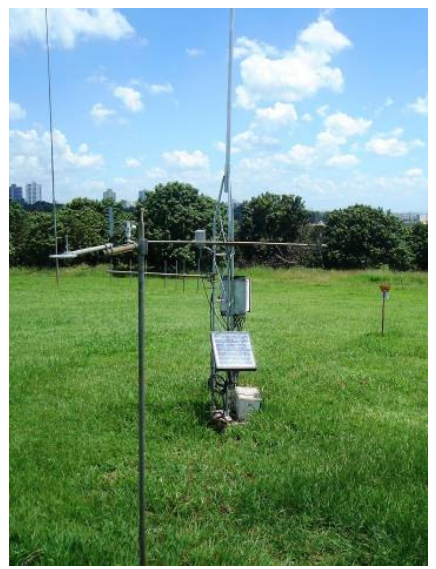

a

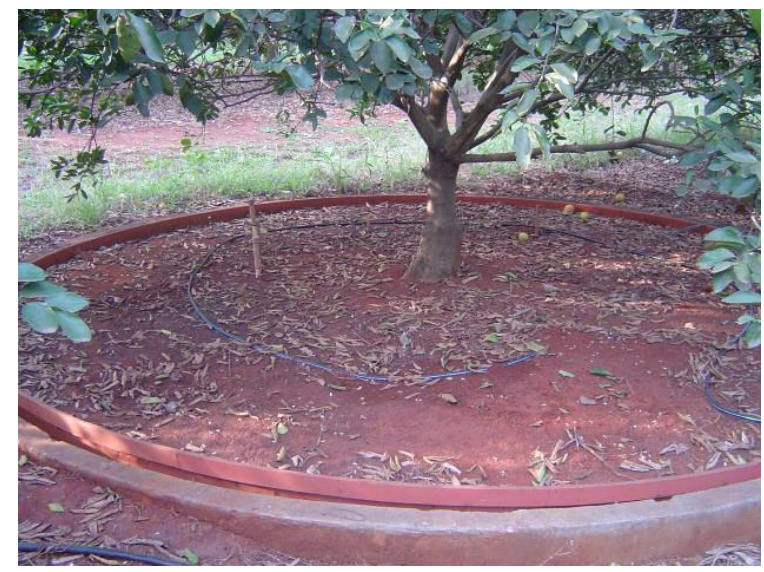

b

FIGURA 1. Estação meteorológica automática e pomar ao fundo (a); lisímetro de pesagem de $4 \mathrm{~m}$ de diâmetro contendo uma planta de limão Tahiti (b). Automatic meteorological station and orchard behind (a); lysimeter of weighting of $4 \mathrm{~m}$ of diameter containing a lemon plant Tahiti (b).

Para a aferição de que o crescimento da planta do lisímetro era similar às demais plantas do pomar, foi realizada uma avaliação fitométrica no final do período de inverno (20-9-2007), consistindo de medidas da altura, perímetro do caule $(0,05 \mathrm{~m}$ acima do ponto de enxertia) e diâmetro da copa da planta do lisímetro e em 10 plantas externas, escolhidas aleatoriamente (Tabela 1).

TABELA 1. Medidas fitométricas da planta cultivada no lisímetro e de plantas cultivadas na área. Measures of the plant cultivated in the lysimeter and of plants cultivated in the área.

\begin{tabular}{|c|c|c|c|c|c|}
\hline \multirow[t]{2}{*}{ Planta } & Altura & $\begin{array}{c}\text { Perímetro }{ }^{1} \text { do } \\
\text { Caule }\end{array}$ & $\begin{array}{c}\text { Diâmetro da } \\
\text { Copa }\end{array}$ & $\begin{array}{c}\text { Área Sombreada } \\
\text { pela Copa }\end{array}$ & $\begin{array}{c}\text { Grau de Cobertura } \\
\text { do Solo }\end{array}$ \\
\hline & & $(\mathrm{m})$ & & $\left(\mathrm{m}^{2}\right)$ & $(\%)$ \\
\hline Lisímetro & 4,00 & 0,52 & 4,98 & 19,92 & 71,14 \\
\hline Pomar $^{2}$ & 4,09 & 0,55 & 4,86 & 19,46 & 69,49 \\
\hline
\end{tabular}

${ }^{\mathrm{T}}$ Medida 0,05 $\mathrm{m}$ acima do ponto de enxertia; ${ }^{2}$ Valores médios de 10 plantas.

\section{Evapotranspiração da cultura (ETc) e coeficiente de cultivo $(\mathrm{Kc})$}

A evapotranspiração da cultura (ETc) foi estimada dividindo-se o total de água consumida pela planta, pela área total $28 \mathrm{~m}^{2}(7 \mathrm{~m} \mathrm{x} 4 \mathrm{~m})$, alocada para cada planta [eq.(1)]. 


$$
\mathrm{ETc}=\frac{\left[\left(\mathrm{M}_{\mathrm{i}-1}-\mathrm{M}_{\mathrm{i}}\right)-\mathrm{D}+\mathrm{I}+\left(\mathrm{P} \mathrm{A}_{\mathrm{L}}\right)\right]+\left(\text { Ke ETo } \mathrm{A}_{\mathrm{E}}\right)}{\mathrm{A}_{\mathrm{T}}}
$$

em que,

ETc - evapotranspiração da cultura, mm;

$\mathrm{M}_{\mathrm{i}-1}$ - massa do lisímetro no dia anterior, $\mathrm{kg}$;

$\mathrm{M}_{\mathrm{i}}$ - massa do lisímetro atual, $\mathrm{kg}$;

$\mathrm{D}$ - drenagem, $\mathrm{kg}$;

$\mathrm{P}$ - precipitação efetiva sobre o lisímetro, $\mathrm{mm}$;

I - irrigação, kg;

$\mathrm{A}_{\mathrm{L}}$ - área do lisímetro, $12,56 \mathrm{~m}^{2}$;

$\mathrm{Ke}$ - coeficiente de evaporação do solo na área externa, adimensional;

ETo - evapotranspiração de referência, $\mathrm{mm} \mathrm{dia}^{-1}$;

$\mathrm{A}_{\mathrm{E}}$ - área externa ao lisímetro, $15,44 \mathrm{~m}^{2}$, e

$\mathrm{A}_{\mathrm{T}}$ - área alocada para cada planta, $28 \mathrm{~m}^{2}$.

O Ke foi estimado por uma curva de evaporação como função de dias após a chuva, conforme descrito por SILVA (2005), ALVES JÚNIOR (2006), ALVES JÚNIOR et al. (2007) e BARBOZA JÚNIOR (2007).

$$
\mathrm{Ke}=1,26 \mathrm{~d}^{-0,63}
$$

em que,

d - dias após o molhamento.

$\mathrm{O} \quad \mathrm{Kc}$ foi calculado dividindo-se a evapotranspiração da cultura [eq.(1)] pela evapotranspiração de referência estimada por Penman-Monteith (ALLEN et al., 1998), como segue:

$$
\mathrm{Kc}=\frac{\mathrm{ETc}}{\mathrm{ETo}}
$$

\section{RESULTADOS E DISCUSSÃO}

\section{Evapotranspiração da cultura (ETc) e coeficiente de cultivo (Kc)}

Apresentam-se, na Tabela 2, os dados de evapotranspiração de referência (ETo) e de evapotranspiração da cultura (ETc) para o período estudado. Observa-se que os maiores valores de ETo máxima foram registrados nos meses de abril e setembro, alcançando 4,72 e 5,63 mm, respectivamente. Essa tendência decrescente de abril para o mês de junho e posterior retomada de valores maiores é provavelmente relacionada à queda de temperatura do ar e da radiação solar no início do inverno, conforme demonstra a Figura 2.

Observa-se, também, tendência de menor consumo de água pela planta nos meses mais frios, indicando relação bastante estreita entre consumo de água, radiação solar (Figura 2) e temperatura do ar (Figura 3a).

A temperatura também determina variáveis climatológicas, como a pressão de saturação de vapor no ar e influencia no déficit de pressão de vapor (DPV), o qual, por sua vez, tem grande influência na regulação estomática de espécies cítricas e no seu consumo de água. Na Figura 3b, pode-se observar a correlação entre Kc e DPV e verificar tendência de queda do Kc quão maior seja o DPV. 
TABELA 2. Médias mensais da estimativa diária da evapotranspiração de referência (ETo) e da evapotranspiração da cultura (ETc) de lima-ácida 'Tahiti'. Monthly averages of the daily estimate of the reference evapotranspiration (ETo) and of the evapotranspiration of the culture (ETc) of acid lime 'Tahiti'.

\begin{tabular}{|c|c|c|c|c|c|c|c|c|c|}
\hline \multirow{2}{*}{ Mês } & \multicolumn{4}{|c|}{$\mathrm{ETo}^{*}\left(\mathrm{~mm} \mathrm{dia}^{-1}\right)$} & \multicolumn{4}{|c|}{$\mathrm{ETc}^{*}\left(\mathrm{~mm} \mathrm{dia}^{-1}\right)$} & \multirow{2}{*}{$\mathrm{Kc} * *$} \\
\hline & Máx. & Mín. & Média** & Desvio-Padrão & Máx. & Mín. & Média** & Desvio-Padrão & \\
\hline abril & 4,72 & 1,15 & 3,55 & 0,81 & 5,60 & 2,13 & 4,08 & 0,87 & 1,18 \\
\hline maio & 3,46 & 1,15 & 2,64 & 0,60 & 3,45 & 1,76 & 2,75 & 0,48 & 1,08 \\
\hline junho & 3,20 & 1,36 & 2,48 & 0,40 & 3,98 & 1,43 & 2,33 & 0,48 & 0,96 \\
\hline julho & 3,77 & 1,09 & 2,39 & 0,69 & 4,35 & 1,20 & 2,27 & 0,62 & 1,00 \\
\hline agosto & 4,32 & 2,11 & 3,22 & 0,63 & 3,40 & 2,08 & 2,60 & 0,30 & 0,82 \\
\hline setembro & 5,63 & 2,45 & 3,99 & 0,68 & 5,11 & 1,30 & 3,33 & 0,64 & 0,84 \\
\hline Média & 4,18 & 1,55 & 3,05 & 0,64 & 4,32 & 1,65 & 2,89 & 0,57 & 0,98 \\
\hline
\end{tabular}

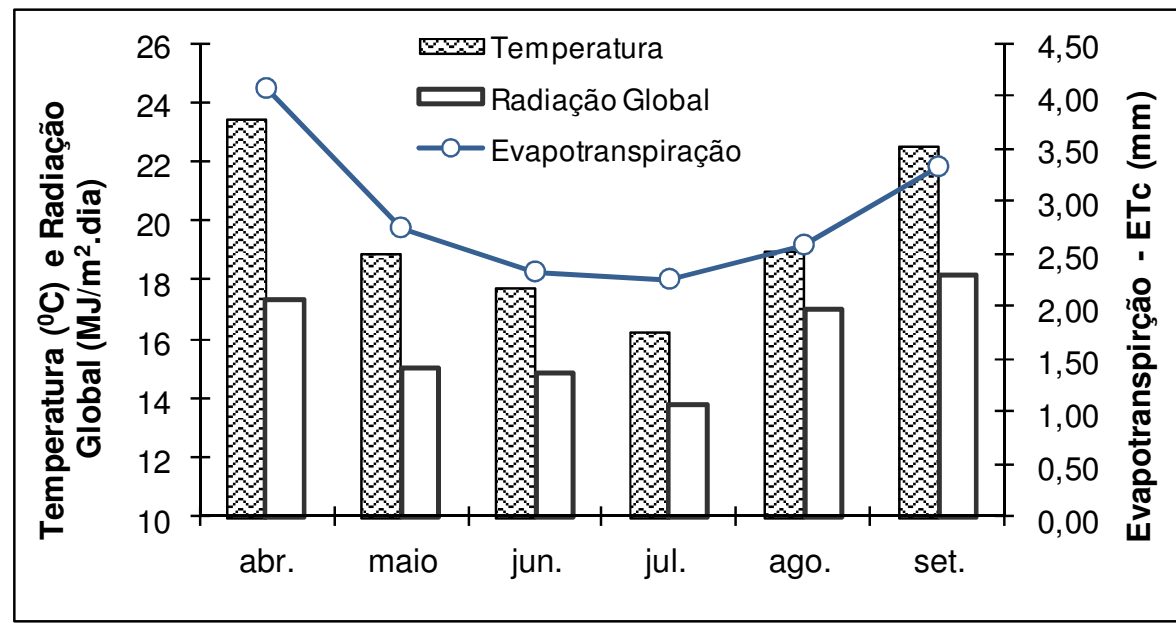

FIGURA 2. Temperatura, radiação global e evapotranspiração de uma planta adulta de lima-ácida Tahiti. Temperature, global radiation and evapotranspiration of an adult plant of acid lime Tahiti.

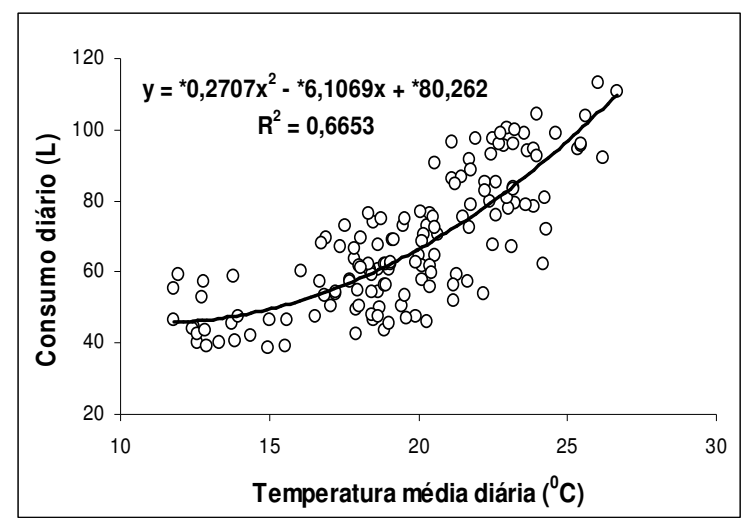

a

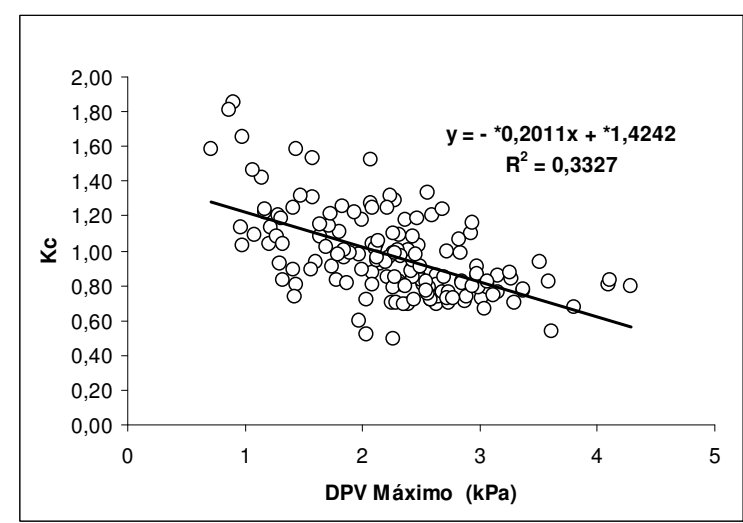

$\mathrm{b}$

FIGURA 3. Temperatura média diária x consumo de água pela planta do lisímetro (a). Coeficiente de cultivo x déficit de pressão de vapor (b). Dados considerando somente dias sem chuva. * Termo significativo a $1 \%$ de probabilidade, pelo teste F. Daily medium temperature vs consumption of water for the plant of the lysimeter (a). Crop coefficient vs deficit of steam pressure (b). Data considering only days without rain. * Significant term to $1 \%$ of probability for the test $\mathrm{F}$. 
De acordo com SYVERTSEN \& LLOYD (1994), o fechamento dos estômatos ocorre durante o aumento do déficit de pressão de vapor folha-ar e representa adaptação evolutiva das espécies cítricas para conservar água.

Em estudo de variabilidade sazonal de variáveis meteorológicas, IRIGOYEN et al. (2007) encontraram que a variação relativa do déficit de pressão de vapor apresentou-se como a principal condicionante das variações da condutância estomática na região de Piracicaba - SP.

YANG et al. (2003), trabalhando com plantas de laranja 'Murcote' sob condições de estufa, mostraram valores de ETc variando de $4,4 \mathrm{~mm} \mathrm{dia}^{-1}$, no verão, a $0,6 \mathrm{~mm} \mathrm{dia}{ }^{-1}$, no inverno, porém relacionados com a área do lisímetro $\left(1,767 \mathrm{~m}^{2}\right)$. Em estudo semelhante, CASTEL (1997), utilizando lisímetro de pesagem com plantas de laranja 'Clementina', encontrou que a evapotranspiração relacionada ao espaçamento de plantio $\left(23,1 \mathrm{~m}^{2}\right)$ variou de $0,7 \mathrm{~mm} \mathrm{dia}{ }^{-1}$, no inverno, a 1,8 e 2,0 $\mathrm{mm} \mathrm{dia}^{-1}$, no verão. Essa tendência também foi observada por SILVA (2005), trabalhando com plantas jovens de lima-ácida Tahiti, com 8\% de grau de cobertura, em Piracicaba - SP, verificando que houve padrão sazonal da evapotranspiração, tendo a média de ETc variando entre $1,5 \mathrm{~mm} \mathrm{dia}^{-1}$, no verão, e $0,66 \mathrm{~mm} \mathrm{dia}^{-1}$, no inverno.

Os valores médios mensais de Kc para plantas adultas com grau de recobrimento médio de $70 \%$ para o período de inverno (estação seca) variaram de 0,82 a 1,18, ficando, na média, em 0,98. Esses valores estão condizentes com os valores apresentados por ALVES JÚNIOR (2006), que mostrou valores médios de coeficiente de cultura para a lima-ácida Tahiti de 0,45;0,56 e 0,68, quando as plantas apresentavam grau de cobertura médio de 9; 20 e 35\%, respectivamente, todos para o período do inverno. Já para o período de verão, o mesmo autor encontrou valores médios de Kc de 0,78; 0,93 e 1,18, quando as plantas apresentavam grau de cobertura médio de 15; 28 e 40\%, respectivamente, em Piracicaba - SP.

VIEIRA \& RIBEIRO (1993), estudando planta adulta lima-ácida 'Tahiti' enxertada em limão-cravo em São Paulo, mostraram valores de Kc de 0,8. Isso provavelmente se deve às diferenças entre variedades, porta-enxerto, espaçamento, idade, solo e método de medida.

BOMAN (1994) relatou que os valores de Kc obtidos via lisimetria na Flórida, em plantas com 3-4 anos de idade, ajustados para área de $28 \mathrm{~m}^{2}$, foram de 0,19 durante o inverno e de 0,34 no verão, enfatizando que pesquisas regionalizadas devem ser desenvolvidas com o intuito de obter valores de Kc locais para o manejo da irrigação.

BERTONHA (1997) estimou Kc de 0,75 para plantas de laranja 'Pera' enxertada em limão 'Cravo' de 4 anos de idade, e Kc igual a 1,0 para laranja 'Baianinha' e 'Hamlin' enxertada em laranja 'Caipira' e limão 'Cravo', respectivamente.

ALLEN et al. (1998), no Boletim da FAO-56, recomendam valores de Kc para citros entre 0,70 e 0,75 para diferentes estádios fenológicos quando o grau de cobertura é em torno de $70 \%$ e a altura de plantas é no máximo $4 \mathrm{~m}$, podendo chegar a valores de até 1,2, dependendo da frequência e do manejo da irrigação. Para as condições onde o experimento foi desenvolvido, utilizando-se da metodologia para ajuste de Kc proposto no próprio Boletim da FAO-56, os valores corrigidos pelas condições meteorológicas locais variam de 0,66 a 0,74 , ficando, portanto, subestimados para o período de menor demanda pelas plantas.

Dados de coeficiente de cultivo de limão geralmente diferem do Kc de laranja, provavelmente porque plantas de limão apresentam maior ETc (cerca de 10 a 20\%), comparado a outros citros, pois essas apresentam crescimento e produção durante todo o ano, como descrito por DOORENBOS \& PRUITT (1977), MORTON (1987) e WRIGHT (2000), citados por ALVES JÚNIOR (2006).

Considerando que em limeiras-ácidas cultivadas em clima tropical não há predominância de estádio fenológico ao longo do ano, pode-se inferir que, em média, em torno de 30 a $50 \%$ do volume de água aplicado pela irrigação no inverno pode ser reduzido, comparado ao período do verão. 


\section{CONCLUSÕES}

A evapotranspiração da cultura (ETc) média no período outono/inverno foi de 2,89 $\mathrm{mm}$.

O coeficiente de cultivo $(\mathrm{Kc})$ médio foi de 0,98 para planta com seis anos de idade.

\section{AGRADECIMENTOS} concedida.

À Fundação de Amparo à Pesquisa do Estado de São Paulo, pela bolsa de estudos

\section{REFERÊNCIAS}

ALLEN, R.G.; PEREIRA, L.S.; RAES, D.; SMITH, M. Crop evapotranspiration: guidelines for computing crop water requirements. 1998. FAO, Rome. In: WRIGHT, G.C. Irrigating citrus trees. 2000. University of Arizona. College of Agriculture. Cooperative Extension. 5 p. Disponível em: $<$ www.cals.arizona.edu/pubs/crops/az1 151.pdf $>$. Acesso em: 15 out. 2007. (Irrigation and Drainage Paper, 56)

ALVES JÚNIOR, J. Necessidade hídrica e resposta da cultura de lima-ácida 'Tahiti' a diferentes níveis de irrigação. 2006. 100 f.Tese (Doutorado em Irrigação e Drenagem) - Escola Superior de Agricultura "Luiz de Queiroz”, Universidade de São Paulo, Piracicaba, 2006.

ALVES JÚNIOR, J.; FOLEGATTI, M.V.; PARSONS, L.R.; BANDARANAYAKE, W.; SILVA, C.R. da; SILVA, T.J.A. da; CAMPECHE, L.F.S.M. Determination of the crop coefficient for grafted 'Tahiti' lime trees and soil evaporation coefficient of Rhodic Kandiudalf clay soil in São Paulo, Brazil. Irrigation Science, Berlin, v.25, n.4, p.419-28, 2007.

BARBOZA JÚNIOR, C.R.A. Evapotranspiração da lima-ácida 'Tahiti' (Citrus latifolia Tan.) determinada por lisimetria de pesagem. 2007. 65 f. Dissertação (Mestrado em Irrigação e Drenagem) - Escola Superior de Agricultura "Luiz de Queiroz", Universidade de São Paulo, Piracicaba, 2007.

BARROS, S.A.; RODRIGUES, J.D.; RODRIGUES, S.D. Efeito do ácido giberélico e do uniconazole na fisiologia pós-colheita do limão 'Tahiti' (Citrus latifolia Tanaka). Revista Brasileira de Fruticultura, Jaboticabal, v.13, n.3, p.223-6, 1991.

BERTONHA, A. Funções de resposta da laranja à aplicação de água e nitrogênio. 1997. 62 f. Tese (Doutorado em Irrigação e Drenagem) - Escola Superior de Agricultura "Luiz de Queiroz", Universidade de São Paulo, Piracicaba, 1997.

BOMAN, B.J. Evapotranspiration from young Florida flatwoods citrus trees. Journal of Irrigation and Drainage Engineering, New York, v.120, n.1, p.81-8, 1994.

CAMPECHE, L.F.S.M. Construção, calibração e análise de funcionamento de lisímetros de pesagem para determinação da evapotranspiração da cultura da lima-ácida 'Tahiti'(Citrus latifolia Tan.). 2002. 62 f. Tese (Doutorado em Irrigação e Drenagem) - Escola Superior de Agricultura "Luiz de Queiroz”, Universidade de São Paulo, Piracicaba, 2002.

CASTEL, J.R. Evapotranspiration of a drip-irrigated clementine citrus trees in a weighing lysimeter. Acta Horticulturae, Leuven, n.449, p.91-8, 1997.

CASTEL, J.R. Response of young clementine citrus trees to drip irrigation. I. Irrigation amount and number of drippers. Journal of Horticultural Science, Kent, v.69, n.3, p.481-9, 1994.

EMBRAPA. EMPRESA BRASILEIRA DE PESQUISA AGROPECUÁRIA. Sistema brasileiro de classificação de solos. Brasília, 1999. 412 p.

HOWELL, T.A.; SCHNEIDER, A.D.; JENSEN, M.E. History of lysimeter design and use for evapotranspiration measurements. In: ALLEN, R.G.; HOWELL, T.A.; PRUITT, W.O., WALTER, 
I.A.; JENSEN, M.E. (Ed.). Lysimeter for evapotranspiration and environmental measurements. New York: American Society of Civil Engineers, 1991. p.1-9.

IRIGOYEN, A.I.; ANGELOCCI, L.R.; CASAROLI, D. Variabilidade horária e sazonal das variáveis meteorológicas determinantes da condutância estomática em Piracicaba - SP. In: CONGRESSO BRASILEIRO DE AGROMETEOROLOGIA, 15., 2007, Aracaju. Anais... Aracaju: Sociedade Brasileira de Agrometeorologia, 2007. 1 CD-ROM.

RAIJ, B. van; SILVA, N.M.; BATAGLIA, O.C.; QUAGGIO, J.A.; HIROCE, R.; CANTARELLA, H.; BELLINAZZI, J.R.; DECHEN, A.R.; TRANI, P.E. Recomendações de adubação e calagem para o Estado de São Paulo. Campinas: IAC, 1992. 107 p. (Boletim Técnico, 100).

SEDIYAMA, C.G.; RIBEIRO, A.; LEAL, B.G. Relações clima-água-planta. In: FARIA, M.A. (Coord.). Manejo de irrigação. Poços de Caldas: UFLA/SBEA, 1998. p.46-85.

SILVA, C.R. Evapotranspiração e desenvolvimento de Limeira Ácida 'Tahiti' na ausência e presença de estresse hídrico. 2005. 85 f. Tese (Doutorado em Irrigação e Drenagem) - Escola Superior de Agricultura “Luiz de Queiroz”, Universidade de São Paulo, Piracicaba, 2005.

SILVA, F.C.; FOLEGATTI, M.V.; MAGIOTTO, S.R. Análise do funcionamento de um lisímetro de pesagem com célula de carga. Revista Brasileira de Agrometeorologia, Santa Maria, v.7, n.1, p.53-8, 1999.

SYVERTSEN, J.P.; LLOYD, J.J. Cítrus. In: SCHAFFER, B.; ANDERSEN, P.C. (Ed.) Handbook of environmental physiology of fruits crops: Subtropical and tropical crops. Boca Raton: CRC Press, 1994. v.2, p.65- 99.

VIEIRA, D.B.; RIBEIRO, T.A.P. Estudo de parâmetros básicos de irrigação do limão 'Tahiti' (Citrus limonia OSBECK) sob microaspersão. In: CONGRESSO BRASILEIRO DE ENGENHARIA AGRÍCOLA, 19., 1993, Ilhéus. Anais ... Ilhéus: Associação Brasileira de Engenharia Agrícola, 1993. v.4, p.2328-39.

YANG, L.S.; YANO, T.M.A.; LI, X. Evapotranspiration of orange trees in greenhouse lysimeters. Irrigation Science, Berlin, v.21, n.1, p.145-9, 2003. 